expressed in the humanized stromal fat pads, some of the injected human MEC cells that were isolated from mammoplasty samples developed into invasive and poorly differentiated carcinomas. Surprisingly, if MECs taken from the same patient but from different regions of the mammoplasty tissue were used, no carcinoma was formed.

The authors suggest that the reduction mammoplasty MECs must have included cells that were already abnormal when removed from the donor mammary gland (although this was undetectable by histology) and that these cells then thrived in the altered stromal environment. This model provides a new way of studying human breast cancer pathogenesis.

Ezzie Hutchinson

\section{(2) References and links} ORIGINAL RESEARCH PAPER Kuperwasser, C. et al. Reconstruction of functionally normal and malignant human breast tissues in mice. Proc. Natl Acad. Sci. USA 101, 4966-4971

WEB SITE

Robert Weinberg's lab

http://web.wi.mit.edu/weinberg/pub/

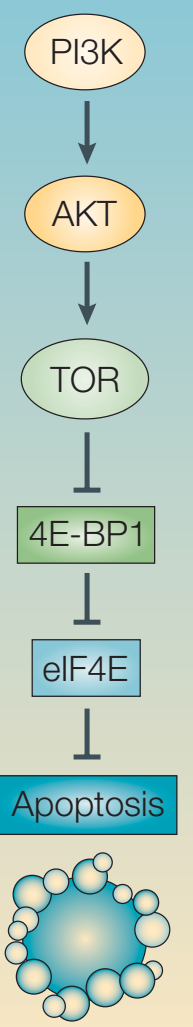

\section{Bullseye}

Developing inhibitors that specifically act on a single protein can be a tricky business, but researchers have hit the bullseye in their attempt to target the insulin-like growth factor receptor-1 (IGF1R). In Cancer Cell, Hofmann and colleagues and Kung and colleagues report that IGF1R inhibitors are selective and have significant antitumour activity.

Insulin-like growth factors and their receptors have been implicated in several processes involved in cancer - proliferation, survival, metastasis and angiogenesis. However, there have been concerns about targeting IGF1R to treat cancer because of its widespread expression in normal tissues and the potential for cross-inhibition of the highly related insulin receptor. Hofmann and colleagues performed a high-throughput screen with their compound archive and identified small molecules of the pyrrolo[2,3- $d]$ pyrimidine class that selectively inhibited IGF1R. They optimized the potency and selectivity of this class - which they assessed using in vitro kinase and cellular assays - and then characterized the most effective, NVP-AEW541. Of particular importance was that this inhibitor was 27 -fold more potent against IGF1R than against the insulin receptor, at the cellular level.

They first showed that NVP-AEW541 inhibited the IGF1-mediated survival of MCF-7 cells and prevented them from growing in the absence of anchorage. It also inhibited the proliferation of NWT-21 cells - this was shown to be through inhibiting the IGF1R kinase and downstream signalling, as the pathway component AKT was no longer phosphorylated and was therefore inactive as a survival protein. In an in vivo model, in which NWT-21 cells were grown subcutaneously in nude mice, oral administration of NVP-AEW541 was able to inhibit tumour growth. So, NVP-AEW541 seems to be a potent and specific anticancer agent that is worth further investigation.

Kung and colleagues started by showing that IGF1R was specifically expressed in haematological and solid cancers, in both cell lines and primary cultures. They then investigated the effects of inhibiting the receptor using several strategies: a neutralizing antibody, a competitive peptide antagonist and a small-molecule inhibitor, NVPADW742, which is similar to NVP-AEW541. All three strategies similarly suppressed the proliferation of serum-stimulated tumour cells, but multiple-myeloma (MM) cells were particularly sensitive. The authors therefore focused on MM, developing an orthotopic xenograft mouse model. NVPADW742 was able to significantly suppress tumour growth and prolong the survival of the mice.

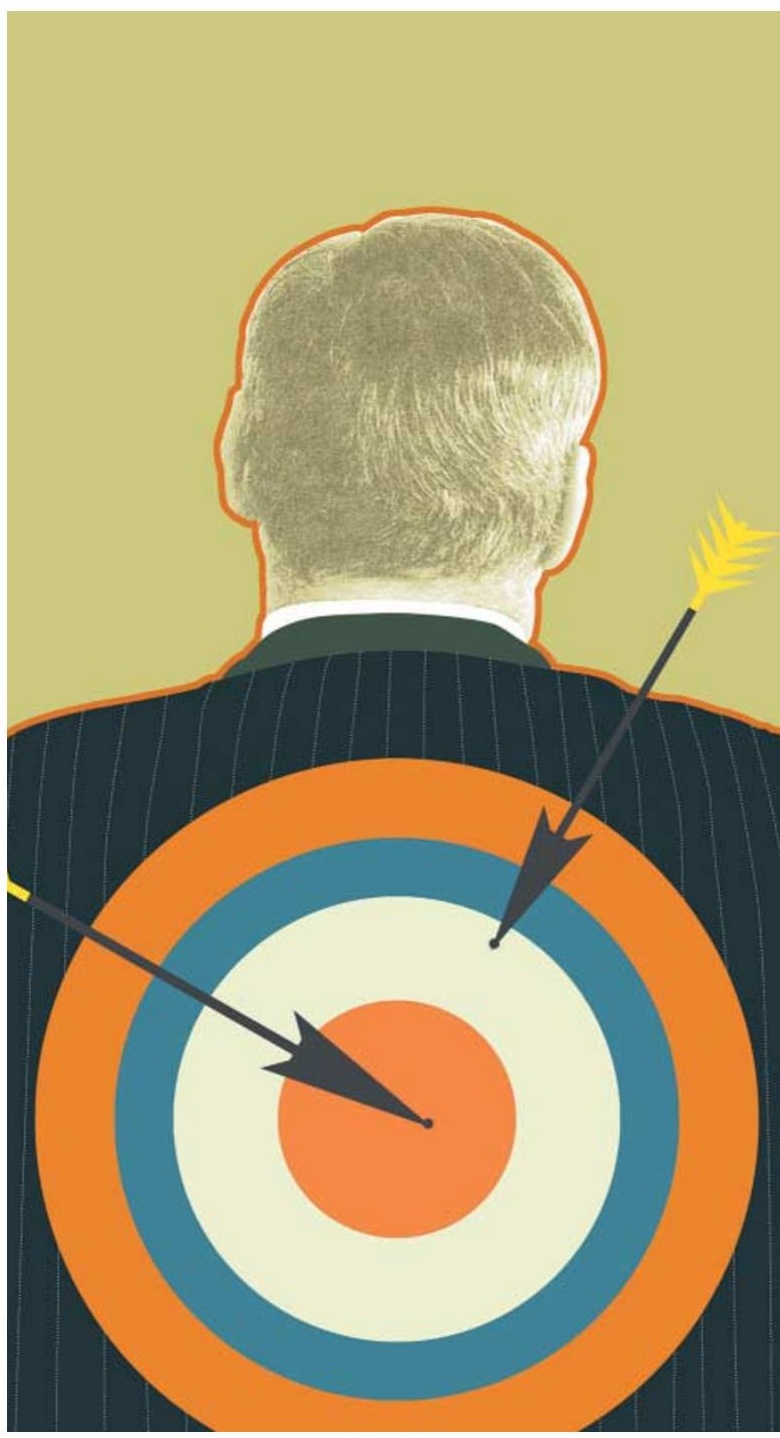

Global expression profiling revealed that inhibition of IGF1R resulted in a highly pleiotropic response, including decreased expression of genes involved in cell-cycle progression, proliferation and survival. This could explain why the inhibitors are effective on a range of tumour types, including those that are resistant to other treatment. Indeed, NVP-ADW742 could sensitize tumour cells to other anticancer agents, such as doxorubicin and melphalan, both in vitro and in vivo.

So, IGF1R inhibitors have been developed that are potent, selective and effective anticancer agents in preclinical models. Pursuing these further should be a useful approach for treating cancer.

Emma Greenwood

\section{(2) References and links}

ORIGINAL RESEARCH PAPERS García-Echeverría, C. et al. In vivo activity of NVP-AEW541 - a novel, potent, and selective inhibitor of the IGF-1R kinase. Cancer Cell 5, 231-239 (2004) | Mitsiades, C. S. et al. Inhibition of the insulin-like growth factor receptor-1 tyrosine kinase as a therapeutic strategy for multiple myeloma, other hematological malignancies, and solid tumors. Cancer Cel/ 5, 221-230 (2004) 